\title{
Untersuchungen von Hammerschlag aus einer Schmiedeesse im römerzeitlichen Marmorsteinbruchrevier Spitzelofen in Kärnten, Österreich
}

\author{
Stephan Karl' ${ }^{1}$ Daniel Modl², Susanne Strobl ${ }^{3}$ und Roland Haubner ${ }^{3}$ \\ ${ }^{1}$ Institut für Antike, Karl-Franzens-Universität Graz, Graz, Österreich \\ ${ }^{2}$ Abteilung Archäologie \& Münzkabinett, Universalmuseum Joanneum, Graz, Österreich \\ ${ }^{3}$ Technische Universität Wien, Wien, Österreich
}

Eingegangen 6. Mai 2021; angenommen 11. Mai 2021; online publiziert 24. Juni 2021

\begin{abstract}
Zusammenfassung: Im römerzeitlichen Marmorsteinbruchrevier Spitzelofen in Kärnten wurden bei archäologischen Ausgrabungen metallurgische Reste aus einer Schmiedeesse gefunden, die die Bearbeitung der Werkzeuge in unmittelbarer Nähe zum Steinbruch belegen. Aus einer holzkohlehaltigen Schicht wurden drei Korngrößenfraktionen von Hammerschlag gewonnen und untersucht. In allen Proben wurden die Eisenoxide Wüstit ( $\mathrm{FeO}), \mathrm{Ma}-$ gnetit $\left(\mathrm{Fe}_{3} \mathrm{O}_{4}\right)$ und Hämatit $\left(\mathrm{Fe}_{2} \mathrm{O}_{3}\right)$ nachgewiesen. In der feinsten Fraktion $(0-0,5 \mathrm{~mm})$ befanden sich eher gleichförmige Hammerschlagpartikel neben größeren Anteilen an Kalzit (Marmor) und Quarz. In den gröberen Fraktionen lag der Hammerschlag plättchenförmig vor. In den Proben konnte neben den Eisenoxiden lokal auch Schlacke und metallisches Eisen nachgewiesen werden.

Das Vorliegen von plättchenförmigem Hammerschlag zeigt, dass dieser durch Verzunderung während des Schmiedevorgangs entstanden ist und bei den verwendeten Schmiedetemperaturen die Schmelzpunkte von Gusseisen und Schlacke nicht erreicht wurden $\left(T<1150^{\circ} \mathrm{C}\right)$.

Für die Nachbearbeitung von groben Bergbauwerkzeugen (Doppelspitzschlägel, Setzkeile usw.) war es vermutlich nicht notwendig, das gesamte Werkzeug auf Schmiedetemperatur zu erwärmen, sondern nur so weit, dass die gewünschte Verformung erreicht werden konnte.
\end{abstract}

Schlüsselwörter: Marmorsteinbruch, Römische Metallverarbeitung, Hammerschlag, Schmieden

\footnotetext{
Prof. Dr. R. Haubner ( $\triangle$ )

Technische Universität Wien,

Getreidemarkt 9/164-03,

1060 Wien, Österreich

roland.haubner@tuwien.ac.at
}

Investigations of Hammerscale from a Forge in the Roman Marble Quarry Area Spitzelofen in Carinthia

Abstract: In the Roman marble quarry area Spitzelofen in Carinthia, the metallurgical remains of a blacksmith's forge were found during archaeological excavations, which proves that tools were worked in the immediate vicinity of the quarry. From a layer containing charcoal, three grainsize fractions of hammerscale were obtained and examined. The iron oxides wüstite $(\mathrm{FeO})$, magnetite $\left(\mathrm{Fe}_{3} \mathrm{O}_{4}\right)$, and hematite $\left(\mathrm{Fe}_{2} \mathrm{O}_{3}\right)$ were detected in all samples. In the finest fraction $(0-0.5 \mathrm{~mm})$, there were more uniform hammerscale particles along with larger proportions of calcite (marble) and quartz. In the coarser fractions, the hammerscale was in the form of platelets. In addition to iron oxides, local slag and metallic iron could be detected in the samples.

The presence of flaky hammerscale shows that this was caused by scaling during the forging process and that the melting points of cast iron and slag were not reached at forging temperature $\left(T<1150^{\circ} \mathrm{C}\right)$.

For the post-processing of coarse mining tools (doublepointed picks, wedges, etc.), it was probably not necessary to heat the whole tool to forging temperature but only to the point where the desired deformation could be achieved.

Keywords: Marble quarry, Roman metal working

Hammerscale, Forging

\section{Archäologisches Umfeld}

Bereits unmittelbar nach der römischen Okkupation Noricums um 16/15 v. Chr. strebten die Römer danach, den weiBen Marmor, den sie in Italien für ihre Gräber und offiziellen Bauten wertzuschätzen gelernt hatten, auch im norischen Ostalpenraum direkt zu erschließen. Schnell gelangten sie 


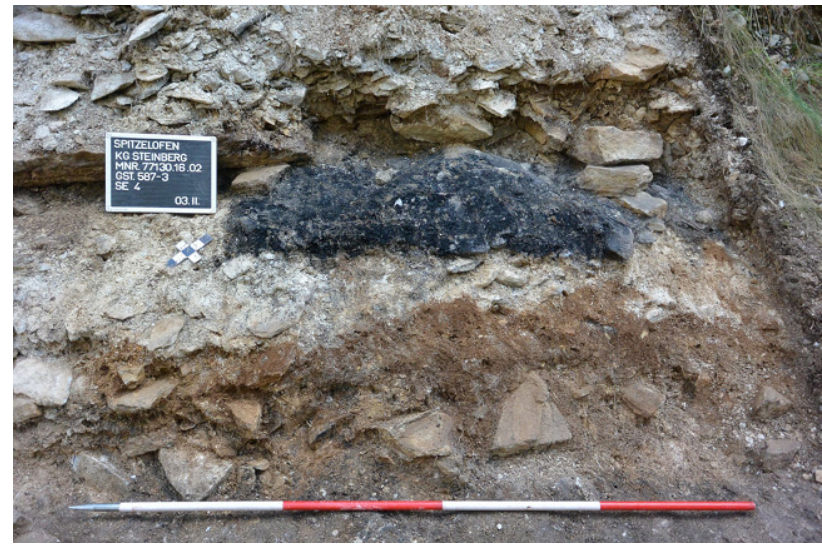

Abb. 1: Holzkohleschicht in der Abraumhalde eines Steinbruchs (GO3) im Marmorsteinbruchrevier Spitzelofen (Foto: Astrid Steinegger)

zu einem Überblick über die geologisch im Ostalpinen Kristallin anstehenden Marmorzüge und -körper, wie im Millstätter Seengebirge, auf der Kor- und Gleinalpe oder am Pohorje [1]. Zu den frühesten Marmorlagerstätten gehören Gummern und Kraig (in der Nähe des norischen Hauptorts am Magdalensberg), die bereits am Beginn des 1. Jahrhunderts n.Chr. ausgebeutet wurden. Diese Kenntnis basiert vorwiegend auf Marmorprovenienzanalysen, d.h. der Bestimmung der Herkunft des Marmors bei römerzeitlichen (datierbaren) Steindenkmalen; so sollen zum Beispiel die Stele der Vettia Prima vom Magdalensberg aus der Zeit um Christi Geburt ([2] http://lupa.at/889) oder die Bauinschrift der Kaiser Augustus und Tiberius aus Emona (Ljubljana) aus den Jahren 14/15 n. Chr. ([2] http://lupa.at/9246) aus Gummerner Marmor bestehen [3].

Das in den letzten Jahren intensiv erforschte Marmorsteinbruchrevier Spitzelofen am Westabhang der Koralpe in Kärnten (Sankt Georgen im Lavanttal, Bezirk Wolfsberg) zählt ebenfalls zu den früh genutzten Marmorlagerstätten. Hier ist jedoch die frühe Erschließung, die durch die analysierte und dem Spitzelofener Marmor zugewiesene Bauinschrift des 69 n. Chr. amtierenden Procurators Petronius Urbicus ([2] http://lupa.at/5769) bislang angenommen wurde, nun auch durch einen archäologischen Befund direkt im Steinbruchrevier nachweisbar [1].

In der Schutthalde eines Steinbruchs (GO 3) im südwestlichen Bereich des Steinbruchreviers Spitzelofen, dem Bruchgebiet Kalkkogel, konnte im Zuge einer Grabung im Jahre 2016 eine kompakte Holzkohleschicht (SE 4), eingebettet zwischen zwei massiven Lagen aus Marmorbruch, freigelegt werden (Abb. 1). Da diese nahezu "reine" Holzkohleschicht von mikrometallurgischen Funden, nämlich Hammerschlag, regelrecht durchsetzt war, ist davon auszugehen, dass es sich dabei um Brennstoffrückstände einer Schmiedeesse beziehungsweise um Abfälle aus dem Bereich der Ambossstelle eines Eisenschmiedehandwerkers handelt. Zusätzlich stammt aus dieser Schicht ein glasig verschlacktes Rotlehm- bzw. Ziegelstück, das als Bestandteil einer Ofen-/Esseneinfassung angesprochen werden kann. Weiters gelangten auch Speisereste (unverbrannte Tierknochen) sowie zerbrochene Gefäßteile aus Keramik in dieses verlagerte Material. Es ist zu vermuten, dass sich die
Schmiedeesse mit der zugehörigen Ambossstelle in unmittelbarer Nähe dieser verlagerten Rückstände befunden hat. Die am ehesten frühkaiserzeitlich zu datierenden Keramikfragmente sowie zwei Holzkohleproben, von denen Radiokarbondatierungen vorliegen, stellen diesen Befund in die Zeit spätestens um die Mitte des 1. Jahrhunderts n. Chr.

Eine vor Ort eingerichtete Schmiede war für den Steinbruchbetrieb unumgänglich, um die verwendeten Eisenwerkzeuge zeitnah reparieren zu können; insbesondere aber, um die stark beanspruchten Doppelspitzschlägel sowie auch Flach-/Spitzmeißel oder Setzschlägel regelmäßig nachzuspitzen. Vom Marmorsteinbruchrevier stammt im Vergleich zu anderen Steinbrüchen eine erstaunlich hohe Anzahl an Steinbruchwerkzeugen, sodass auch die im Marmorabbau verwendeten Werkzeuge beurteilt werden können [1]. Inklusive der drei Altfunde (Grabung 1931) umfasst der Fundkomplex insgesamt 32 vollständig erhaltene Steinbruchwerkzeuge und ein kleines Fragment eines Doppelspitzschlägels mit einem Gesamtgewicht von knapp über $73 \mathrm{~kg}$ (Abb. 2).

Um effektiv im Marmor arbeiten zu können, wie z. B. bei der Anlage von Schrämgräben, braucht es gut gespitzte Werkzeuge, da eine stumpfe Spitze ihre Sprengwirkung verliert und die Kristalle nur mehr zu Marmormehl zerdrückt, das in der Folge dem Schlag dämpfend entgegenwirkt. Experimentelle Untersuchungen zeigen, dass die Meißel alle zwei Stunden ausgewechselt bzw. - am Ende des Arbeitstags - nachgespitzt werden mussten [4]; anderen Berichten zufolge geschah dies mindestens einmal pro Stunde [5]. Neben der Erfahrung und Menschenkraft der Steinbrucharbeiter bestimmten Schmiedehandwerk und insbesondere die Qualität des Eisens die Effektivität des Vortriebs im Marmor. Für die Werkzeuge griff man auf den „norischen Stahl“ (ferrum Noricum) zurück, der im Römischen Reich als Qualitätsprodukt von sprichwörtlicher Härte bekannt war [6, 7]. Bei einem metallurgisch untersuchten Doppelspitzschlägel (Has-12) vom Spitzelofen konnte nachgewiesen werden (Abb. 3), dass dieser weitgehend aus Stahl mit eutektoi$\operatorname{dem}(0,8 \% \mathrm{C})$ und geringeren untereutektoidem $(<0,8 \% \mathrm{C})$ Kohlenstoffgehalt besteht [8]. Die Produktionsstätten des verwendeten Eisens sind nicht belegt, aber die Eisenproduktion selbst erfolgte in sogenannten Rennfeuern [9] und die derart erhaltenen Luppen wurden durch Schmieden zu Werkzeugen umgeformt [10].

\section{Schmiedeessen in antiken Steinbrüchen}

Obwohl die Existenz von Schmiedeessen bei allen Steinbrüchen vorauszusetzen ist, sind archäologisch dokumentierte (und publizierte) Befunde von solchen eher einfach ausgestatteten Feldschmieden im gesamten Römischen Reich selten [11]. Aus den bekannten Marmorlagerstätten bei Carrara (Luni) sind Beobachtungen aus dem frühen 19. Jahrhundert überliefert, die von Mulden mit verbrannter und schwarzer (Holzkohle?) Erde und Schlacken berichten [12-14]. Schlackenfunde, die auf das Vorhandensein von Schmieden in den jeweiligen Steinbrüchen hinweisen, sind weiters in den Marmorsteinbrüchen von Aliki auf 
Abb. 2: Sämtliche Steinbruchwerkzeuge aus dem Marmorsteinbruchrevier Spitzelofen Vorschlaghammer, Doppelspitzschlägel, Setzschlägel, Setzkeile, Brechstangen und Vollschaftmeißel; * Objekte im Museum Lavanthaus in Wolfsberg; ${ }^{+}$der metallurgisch untersuchte Doppelspitzschlägel Has-12 (Grafik: Stephan Karl)
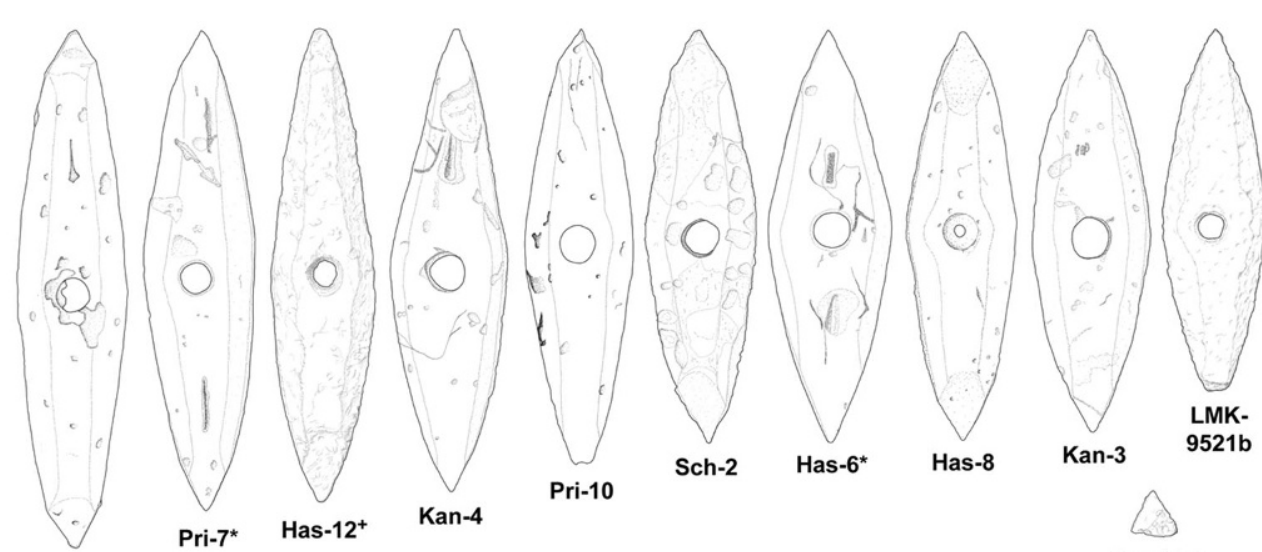

Has-10-3
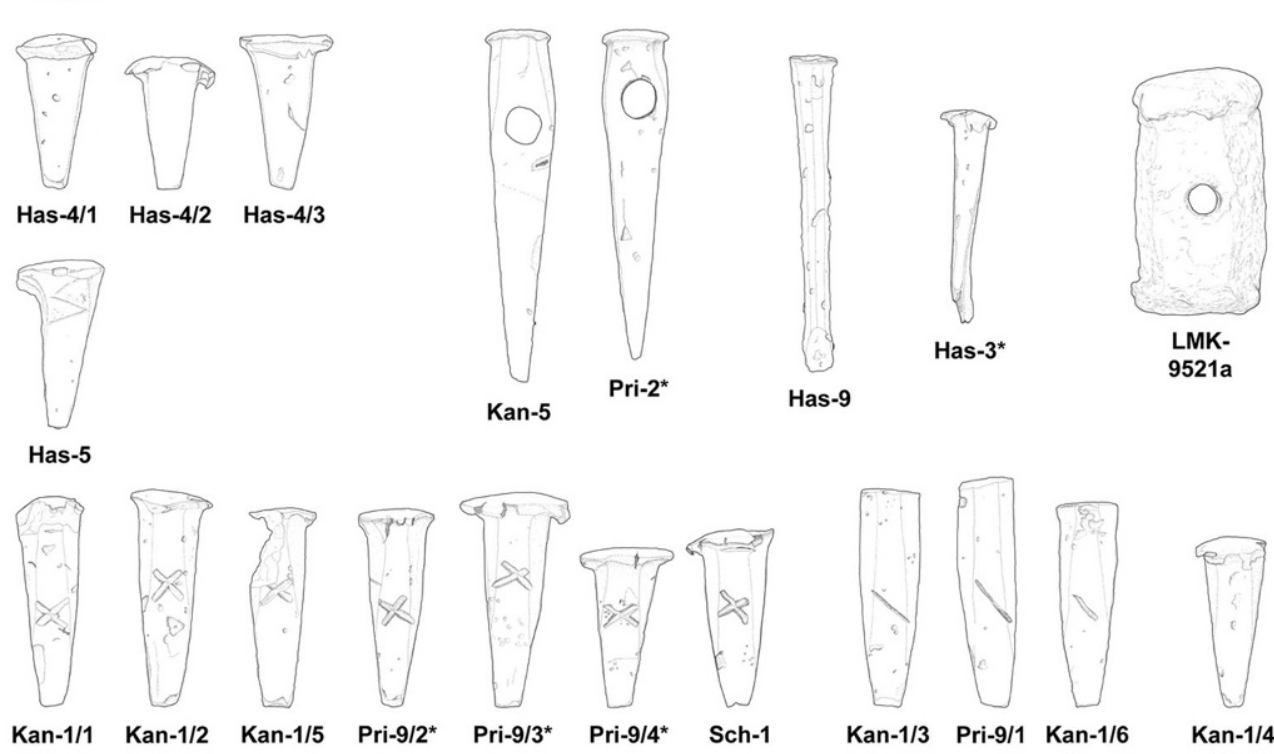

Pri-8*
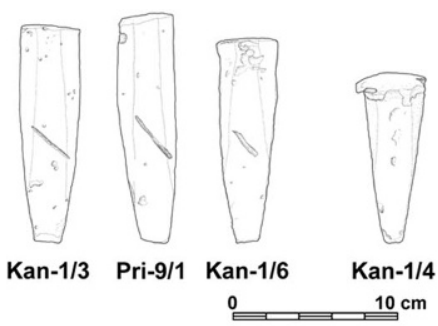

Kan-2

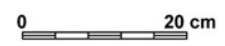

der ägäischen Insel Thasos [15] und von Saint-Béat nahe Saint-Bertrand-de-Comminges in Südfrankreich [16] belegt. Die umfangreichsten Belege zu Schmiedetätigkeiten stammen aus dem unter kaiserlicher Verwaltung stehenden Granit-Steinbruchrevier am Mons Claudianus in der ägyptischen Ostwüste: sowohl archäologisch als auch in Schriftquellen $[5,17]$. Dort wurden in 24 von 130 identifizierten Steinbrüchen Ablagerungen von Asche und Eisenschlacken festgestellt; Schmiedeaktivitäten konnten in vier ausgegrabenen Steinbruchhütten anhand der Herd- und Blasbalgstellen und den diversen Asche-, Holzkohle- und Schlackenschichten nachgewiesen werden; ein gewisser Anfall von Hammerschlag wird im Rahmen der metallurgischen Untersuchung von Freestone erwähnt [17]. Die Bedeutung der Schmiedetätigkeit für den Betrieb eines Steinbruchs geht jedoch am deutlichsten aus den Schriftquellen hervor. Unter den über 6000 Ostraka vom Mons Claudianus werden neben den eigentlichen Steinbrucharbeitern noch spezialisierte Handwerker für das Schmieden (chalkeis), für das Metallhärten (pharmaxarioi) und für den Betrieb des Blasebalgs (physetai) genannt. Aus einzelnen Ostraka lässt sich zum Beispiel ein Verhältnis zwischen Handwerkern, die mit Schmiedetätigkeiten zu verbinden sind, und den eigentlichen Steinbrucharbeitern von 1:4 erschließen; d.h. das "Schmiedeteam“ aus den genannten drei Handwerkern stand einem Steinbrucharbeiter im Verhältnis 1:12 gegenüber [5].

Eine solche Spezialisierung und Arbeitsaufteilung, wie in den kaiserlichen Brüchen von Ägypten, ist natürlich bei den wesentlich kleineren Marmorsteinbruchrevieren im Ostalpenraum, wie am Spitzelofen, natürlich nicht zu erwarten. Hier dürften die Steinbrucharbeiter selbst diese Tätigkeiten des Nachspitzens und Reparierens ihrer Werkzeuge durchgeführt haben; ähnlich wie dies zum Beispiel auch bei der Gewinnung von Schleifsteinen in Sandsteingruben im vorigen Jahrhundert praktiziert wurde [18].

Archäologisch ist jedoch von Bedeutung, dass uns diese Steinbruchschmieden, die auf jeden Fall anhand der 

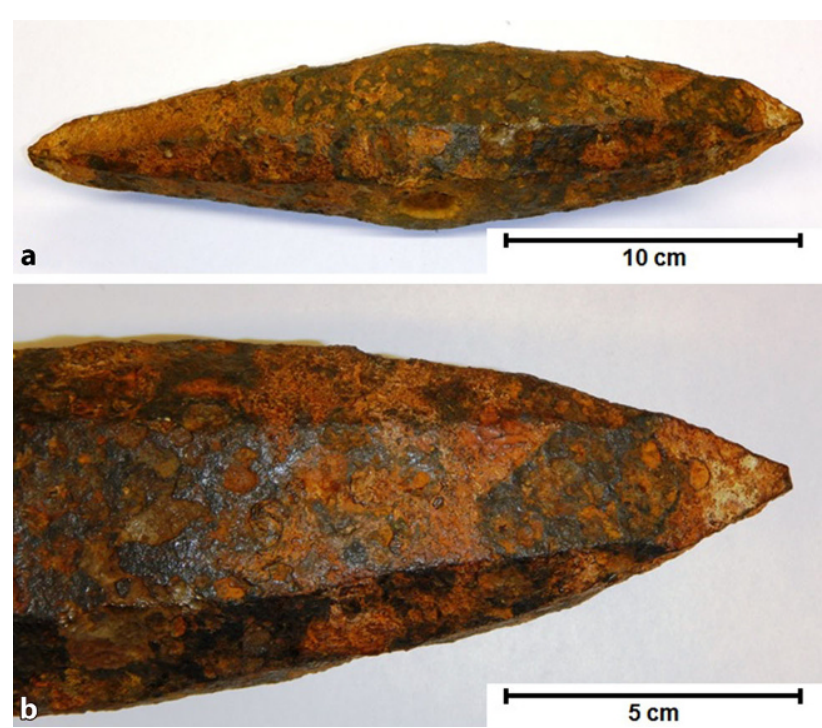

Abb. 3: Der metallurgisch untersuchte Doppelspitzschlägel (Has-12) aus dem Marmorsteinbruchrevier Spitzelofen

Holzkohle mittels Radiokarbondatierung zeitlich näher bestimmt werden können, die einzigartige Möglichkeit geben, auch die betreffende Steinbruchaktivität datieren zu können; Steinbruchwerkzeuge und Abbauspuren - wenn überhaupt vorhanden - wären dafür chronologisch zu unempfindlich.

\section{Schmieden}

Schmieden von Metallen ist wahrscheinlich das älteste Formgebungsverfahren in der Metallurgie und war auch bei den Römern bereits weit entwickelt [7, 10, 19]. Beim Schmieden von Eisen tritt Materialverlust an den MetalIoberflächen durch Oxidation des Metalls unter Einwirken von Luftsauerstoff bei hohen Temperaturen auf. Es entstehen Oxidschichten, die überwiegend aus Magnetit $\left(\mathrm{Fe}_{3} \mathrm{O}_{4}\right)$ und Wüstit $(\mathrm{FeO})$ bestehen, welche beim Schmieden als Eisenoxidplättchen abspringen. Diese Plättchen werden auch als Hammerschlag oder Zunder bezeichnet [20, 21].

Da die Eisenproduktion der Römer in sogenannten Rennfeuern durchgeführt wurde, ist auch die Inhomogenität der erhaltenen Eisenluppen zu berücksichtigen. Diese Luppen haben einerseits eine inhomogene Kohlenstoffverteilung, wobei von reinem Eisen, über Stahl bis zu Gusseisen alle Kohlenstoffgehalte auftreten können [22, 23], andererseits können auch noch Schlackenanteile in der Luppe und später in den geschmiedeten Teilen vorliegen.

Die umfangreichen Untersuchungen von Hammerschlag durch Dungworth und Wilkes [20, 21] können folgendermaßen zusammengefasst werden.

Kugelförmiger Hammerschlag entsteht aus geschmolzenem Material, welcher durch den Schmiedevorgang wegspritzt und im Flug erstarrt. Es wurden Kugeln aus Schlacke oder Gusseisen, aber auch Hohlkugeln sowie Kugeln mit einem Metallkern und einer Oxidschale oder Schlacke gefunden.

Plättchenförmiger Hammerschlag besteht überwiegend aus Eisenoxiden, die teilweise Schlackeneinschlüsse enthalten. Dieser ist auf die Zunderbildung während des Schmiedevorgangs und Abplatzen von der Oberfläche zurückzuführen.

Im geschmiedeten Metall erfolgt durch die Schmiedetemperatur, aber auch durch das Schmieden selbst, eine Homogenisierung der Kohlenstoffverteilung und eine Verformung der Schlackeneinschlüsse [24-26].

\section{Werkstoffkundliche Untersuchungen}

Die verschiedenen Fraktionen der Hammerschlagproben wurden mittels Metallographie, Lichtmikroskop (LOM), Rasterelektronenmikroskop (REM) mit Rückstreuelektronen (BSE) und energiedispersiver Röntgenanalyse (EDX), 3D-Digitalmikroskop (3D-DM) sowie Röntgenbeugung (XRD) untersucht.

Für die Metallographie wurden Pulver in Araldit kalteingebettet und danach geschliffen und poliert.

\section{Untersuchungen des Hammerschlags}

Für die Untersuchungen standen drei Kornfraktionen (0,5-1 mm, 1-2 mm, 2-4 mm) zur Verfügung.

Im 3D-DM erscheint die Kornfraktion 0,5-1 mm körnig und farblich inhomogen (Abb. 4a-c). Um diese Inhomogenitäten abzuklären, wurden XRD-Untersuchungen an den einzelnen Kornfraktionen durchgeführt (Tab. 1). Dabei zeig-

TABELLE 1

Phasenzusammensetzung der Hammerschlagfraktionen nach XRD-Analysen

\begin{tabular}{|c|c|c|c|}
\hline Gew.\% & $0-0,5 \mathrm{~mm}$ & $1-2 \mathrm{~mm}$ & $3-4 \mathrm{~mm}$ \\
\hline Wüstit, FeO & 5,1 & 30,1 & 29,9 \\
\hline Magnetit, $\mathrm{Fe}_{3} \mathrm{O}_{4}$ & 7,3 & 25,3 & 29,7 \\
\hline Hämatit, $\mathrm{Fe}_{2} \mathrm{O}_{3}$ & 8,5 & 10,2 & 17,5 \\
\hline Quarz $\mathrm{SiO}_{2}$ & 15,3 & 11,8 & 7,3 \\
\hline Kalzit, $\mathrm{CaCO}_{3}$ & 63,8 & 22,6 & 15,7 \\
\hline \multicolumn{4}{|c|}{ Verhältnisse (gerundet auf 5) } \\
\hline Wüstit, FeO & 25 & 45 & 40 \\
\hline Magnetit, $\mathrm{Fe}_{3} \mathrm{O}_{4}$ & 35 & 40 & 40 \\
\hline Hämatit, $\mathrm{Fe}_{2} \mathrm{O}_{3}$ & 40 & 15 & 20 \\
\hline
\end{tabular}



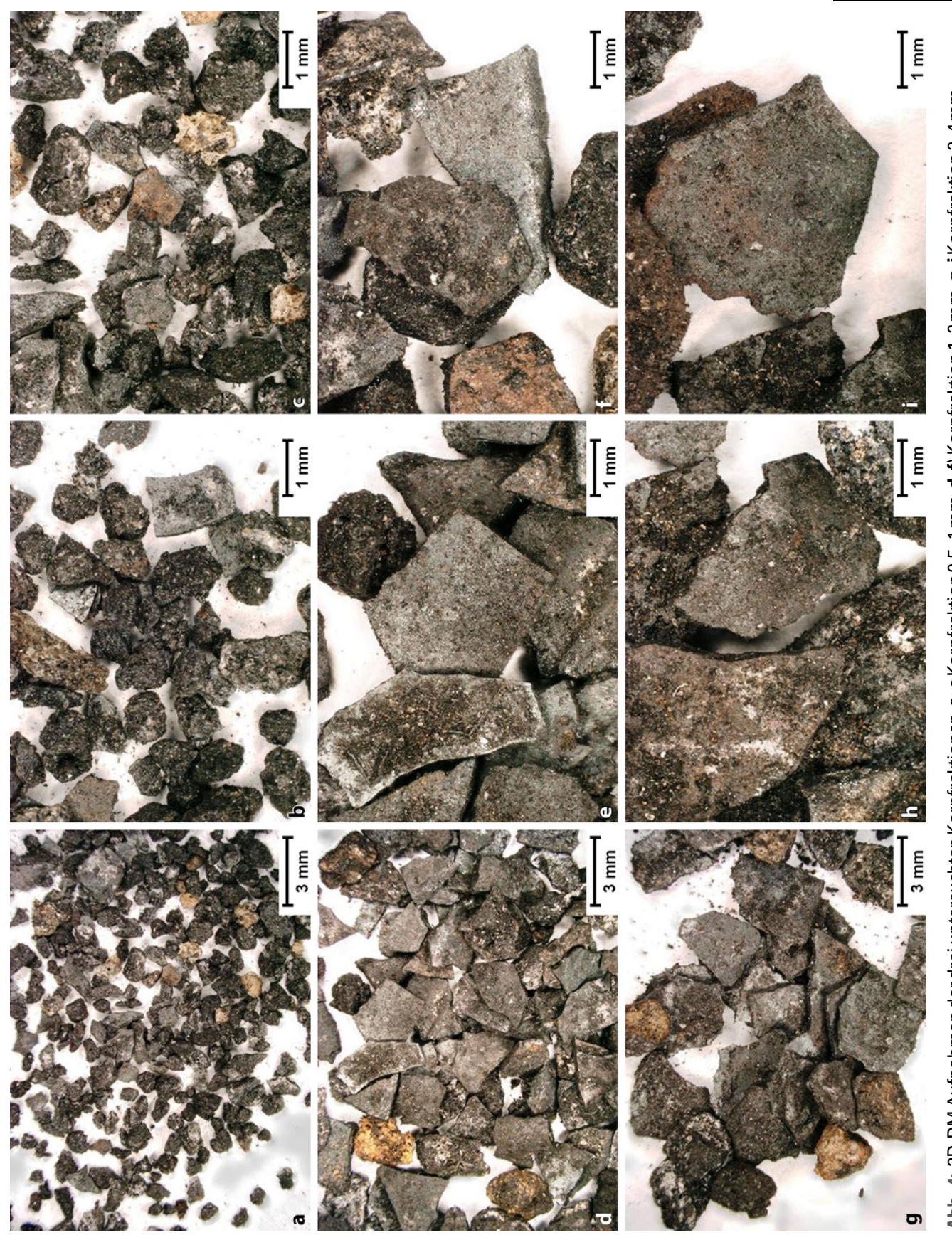

te sich, dass in dieser Fraktion Kalzit (ca. 64 Mass.\%) und Quartz (ca. 15 Mass.\%) neben den Eisenoxiden vorliegen. Der hohe Anteil an Kalzit ist dabei nicht verwunderlich, stammen die Proben doch aus einem Marmorsteinbruch. Weiters wurden die Verhältnisse der Eisenoxide bestimmt $\left(\mathrm{FeO}-\mathrm{Fe}_{3} \mathrm{O}_{4}-\mathrm{Fe}_{2} \mathrm{O}_{3}\right.$ ) (Tab. 1), wobei in der feinsten Kornfraktion die höchsten $\mathrm{Fe}_{2} \mathrm{O}_{3}$ und niedrigsten $\mathrm{FeO}$ Anteile gefunden wurden. Dies könnte dadurch erklärt werden, dass beim Abspringen von kleinen Körnern aufgrund der großen Oberfläche und hohen Temperatur das $\mathrm{FeO}$ rascher zu $\mathrm{Fe}_{3} \mathrm{O}_{4}$ bzw. $\mathrm{Fe}_{2} \mathrm{O}_{3}$ oxidiert wird als bei großen Hammerschlagplättchen.
In der Kornfraktion 1-2 mm (Abb. 4d-f) liegen bereits plättchenförmige Stücke vor, welche Hammerschlag sein könnten, aber auch rundliche Bruchstücke. Die XRD-Untersuchung dieser Kornfraktion (Tab. 1) ergab Kalzit (ca. 23 Mass.\%) und Quartz (ca. 12 Mass.\%), was deutlich weniger ist als in der feineren Fraktion. Bei den Verhältnissen der Eisenoxide untereinander ist in dieser Probe das $\mathrm{FeO}$ am meisten und $\mathrm{Fe}_{2} \mathrm{O}_{3}$ am wenigsten vertreten.

Die Kornfraktion 2-4 mm (Abb. $4 \mathrm{~g}-\mathrm{i}$ ) besteht überwiegend aus Plättchen und nur geringen Anteilen an rundliche Bruchstücke. Die XRD-Messung (Tab. 1) ergab die niedrigsten Werte für Kalzit (ca. 16 Mass.\%) und Quartz (ca. 7 Mass.\%) in den drei Kornfraktionen. 

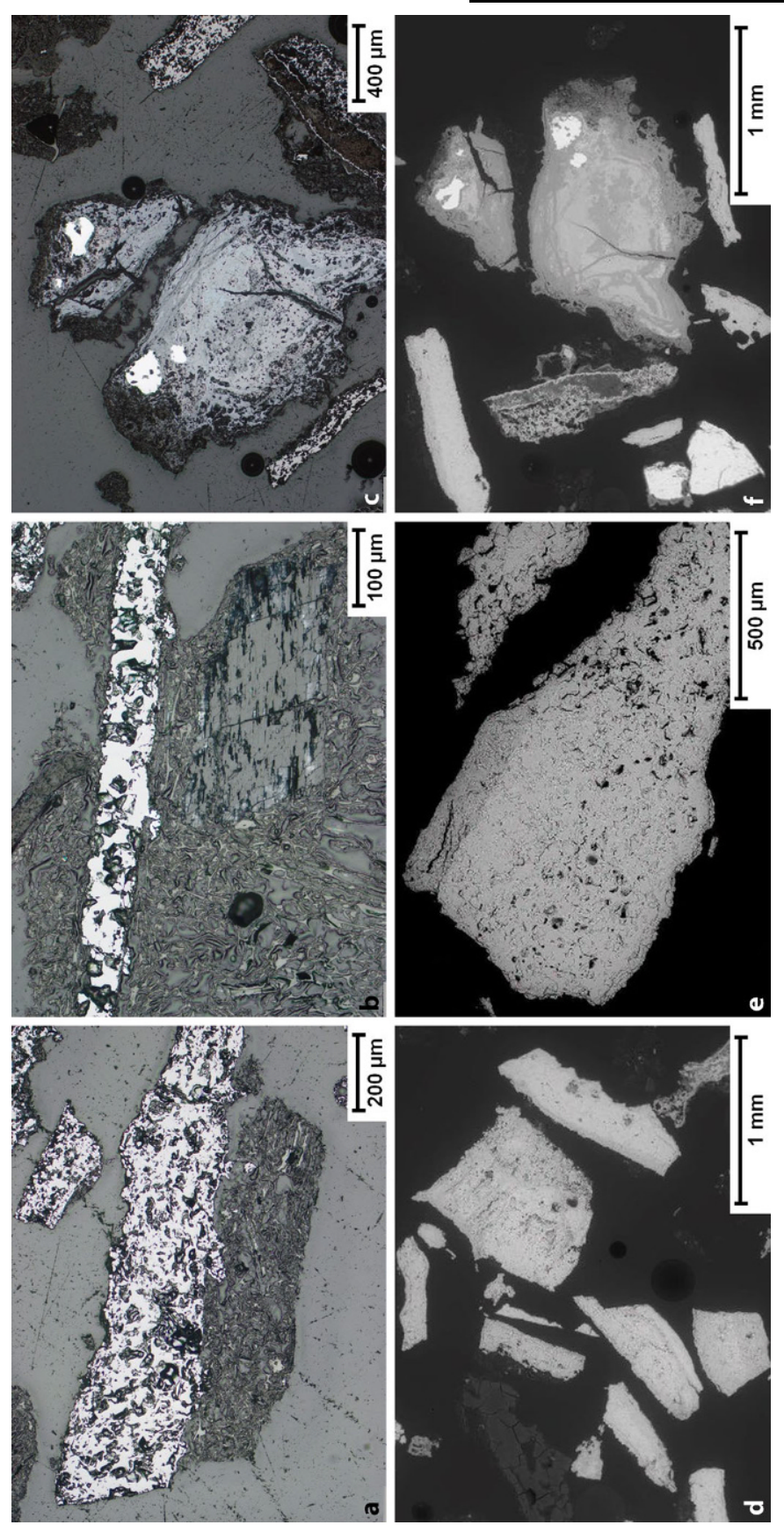

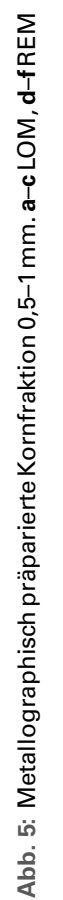

Ergebnisse der metallographisch präparierten Kornfraktionen. Die Verhältnisse der Eisenoxide sind ähnlich wie in der Kornfraktion 2-4 mm.

Im LOM kann gut zwischen Hammerschlag (Eisenoxide bzw. Schlacke) und Holzkohle unterschieden werden. Beispielsweise in Abb. 5a ist der Hammerschlag hellgrau und die Holzkohle dunkelgrau. Die mittelgraue Umhüllung ist das Kalteinbettmittel (Araldit, ein Epoxiharz).

Im REM-BSE ist die Unterscheidung von Holzkohle und Araldit schwierig, da beide überwiegend aus Kohlenstoff bestehen (Abb. $5 \mathrm{~d}$ ). Innerhalb des Hammeschlags sind jedoch unterschiedliche Graustufen zu erkennen. Weiß ent- spricht metallischem Eisen, hellgrau $\mathrm{FeO}$, dunkelgrau $\mathrm{Fe}_{3} \mathrm{O}_{4}$ oder $\mathrm{Fe}_{2} \mathrm{O}_{3}$ und schwarz ist das Einbettmittel.

Bei länglichen Plättchen oder lagiger Anordnung unterschiedlicher Bereiche kann nicht auf die tatsächliche Schichtdicke geschlossen werden, da der Einbettwinkel nicht bekannt ist.

Kornfraktion 0,5-1 mm (Abb. 5) Obwohl in den 3D-DM Bildern körnige Strukturen überwiegen, sind in den Anschliffen (LOM- und REM-Bilder) plättchenförmige Teile zu erkennen, an denen noch Holzkohle anhaftet (Abb. 5a,b). Vereinzelt finden sind auch kleine metallische Eiseneinschlüsse (Abb. 5c,f). Anhand der REM-BSE Bilder erkennt 

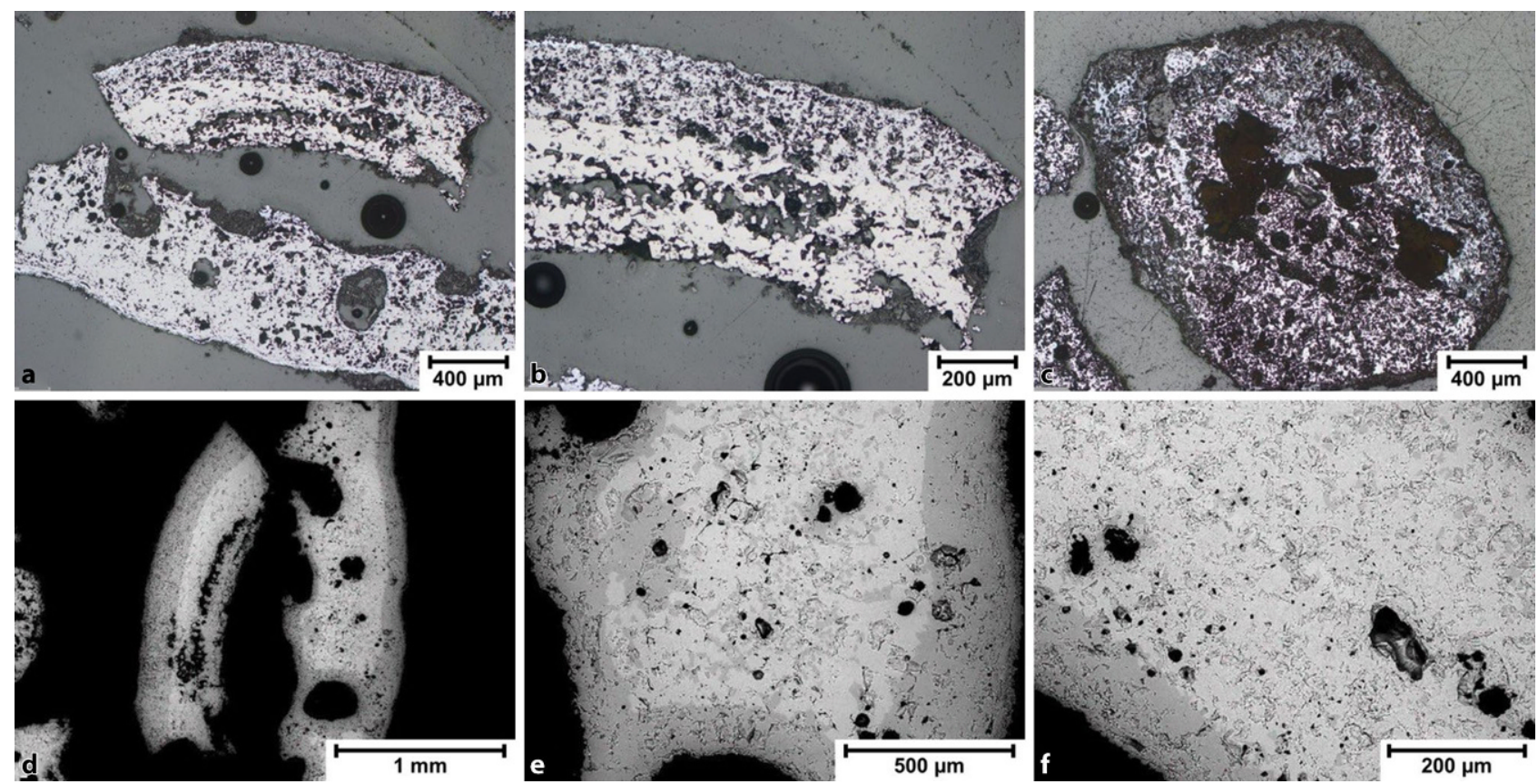

Abb. 6: Kornfraktion 1-2 mm. a-c LOM, d-f REM

man auch den lagigen Aufbau der Hammerschlagplättchen (Abb. 5d,e). In Abb. $5 f$ ist metallisches Eisen (weiß) zu erkennen, jedoch sieht das umgebende Material aus hellgrauen und dunkelgrauen Schlieren nicht wie der kompakte Hammerschlag aus. Die EDX-Analyse ergab überwiegend $\mathrm{Fe}$ und $\mathrm{O}$ mit geringen Konzentrationen an $\mathrm{Ca}$. Vermutlich handelt es sich um korrodiertes Fe also Eisenhydroxide. In sehr inhomogenen Partikeln wurde neben Quarz (Si) auch Ca und $\mathrm{P}$ nachgewiesen.

Kornfraktion 1-2 mm (Abb. 6) Bei dieser Probe sind im Anschliff wiederum Plättchen mit schichtförmigem Aufbau zu sehen (Abb. 6a,b). Die im LOM poröser aussehenden Bereiche können im $\mathrm{REM}$ dem $\mathrm{Fe}_{3} \mathrm{O}_{4}$ bzw. $\mathrm{Fe}_{2} \mathrm{O}_{3}$ zugeordnet werden (Abb. 6d). Über die Entstehung der Poren und Risse in den Schichten kann nur spekuliert werden. Poren könnten ursprünglich metallisches Eisen enthalten haben, welches während der Lagerung korrodiert ist und dadurch einen Hohlraum zurückgelassen hat. Risse sind auf eine Volumensänderungen bei der Bildung unterschiedlicher Eisenoxide zurückzuführen. In Abb. 6e, f sind eher flächige Hammerschlagstücke zu sehen, welche im Zentrum $\mathrm{FeO}$ und an den Rändern $\mathrm{Fe}_{3} \mathrm{O}_{4}$ bzw. $\mathrm{Fe}_{2} \mathrm{O}_{3}$ enthalten. In den feinen Strukturen konnten nur geringe Konzentrationen von $\mathrm{Ca}$, $\mathrm{Si}$ und Al nachgewiesen werden, was darauf hindeutet, dass hier die Verunreinigungen des Eisens in die Oxidschicht eingebaut wurden.

Kornfraktion 2-4 mm (Abb. 7) In dieser gröbsten Fraktion ist der lagige Aufbau des Hammerschlags nicht gut erkennbar und es werden auch vermehrt grobe Löcher beobachtet (Abb. 7a,f,g). Dies könnte auch daran liegen, dass bei der Einbettung von groben Plättchen keine Querschnitte mehr erhalten werden, sondern die Plättchen flach angeschliffen werden. Auffällig ist auch, dass bei dieser Probe vermehrt Schlackenstrukturen beobachtet werden (Abb. 7b,c,g,h,i). In Bereichen, die typische Strukturen von Eisenschlacken aufweisen (Abb. 7g,h), wurde auch Si nachgewiesen, womit hier Fayalit $\left(\mathrm{Fe}_{2} \mathrm{SiO}_{4}\right)$ neben $\mathrm{FeO}$ als Phasen angenommen werden können [27]. Es wurden jedoch auch Bereiche gefunden, in denen neben Fe nur $\mathrm{Ca}$ auftritt (Abb. 7i). Hier dürfte es zu einer Reaktion von gebranntem Marmor ( $\mathrm{CaO})$ mit dem FeO gekommen sein, wodurch neben Mischkristallen (hellgrau) auch Ca-Fe-O Phasen (dunkelgrau) entstanden sind, welche jedoch nicht identifiziert werden konnten [28].

\section{Zusammenfassung}

Der aus der Schmiedeesse im Marmorsteinbruchrevier Spitzelofen gefundene Hammerschlag ist eher plättchenförmig und besteht aus den Eisenoxiden Wüstit ( $\mathrm{FeO})$, Magnetit $\left(\mathrm{Fe}_{3} \mathrm{O}_{4}\right)$ und Hämatit $\left(\mathrm{Fe}_{2} \mathrm{O}_{3}\right)$. Innerhalb des Hammerschlags wurden auch lokal Fayalitschlacke und metallisches Eisen nachgewiesen. Vereinzelt befanden sich CaFe-O Phasen, was auf die Reaktion von Eisenoxiden mit gebranntem Kalk zurückgeführt werden kann. Kugelförmiger Hammerschlag, der durch Wegspritzen von flüssigem Metall oder flüssiger Schlacke entsteht, wurde nicht gefunden. Daraus lässt sich schließen, dass die verwendeten Schmiedetemperaturen unterhalb der Schmelzpunkte von Gusseisen und Schlacke lagen $\left(T<1150^{\circ} \mathrm{C}\right)$.

Plättchenförmiger Hammerschlag entsteht somit durch Diffusion und Oxidbildung (Verzunderung) [29] während des Erhitzens der Werkzeuge und beim Schmiedevorgang kam es zur Abplatzung der spröden Oxidschichten. 

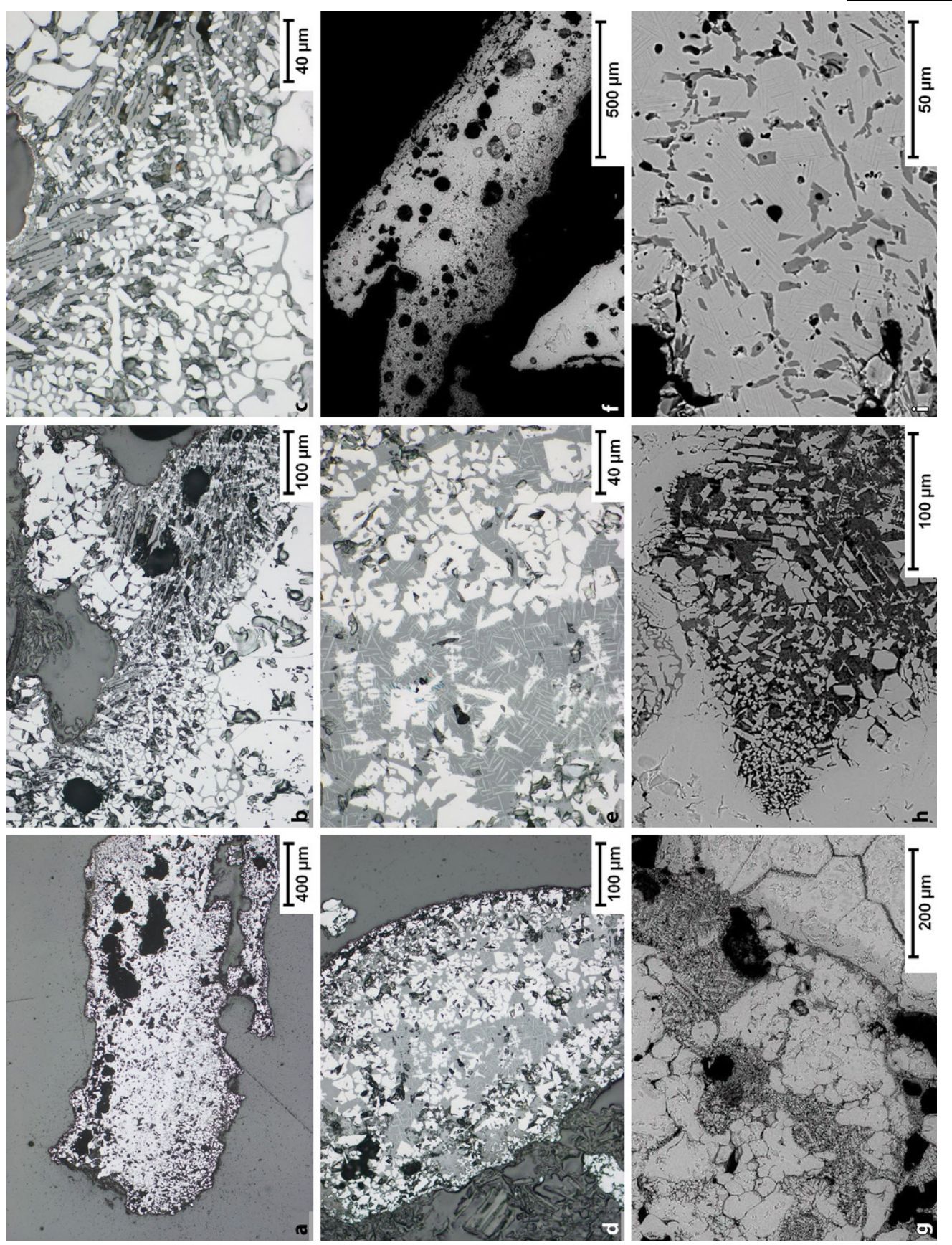

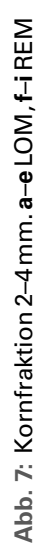

Für die Nachbearbeitung von groben Bergbauwerkzeugen (Doppelspitzschlägel, Setzkeile usw.) war es vermutlich nicht notwendig, das gesamte Werkzeug auf Schmiedetemperatur zu erwärmen, sondern nur so weit, dass die gewünschte Verformung erreicht werden konnte. Es gibt zahlreiche Untersuchungen über die Oxidation von Eisen an Luft und die Ausbildung der Zunderschicht, woraus geschlossen werden kann, dass die Bildung selbst dicker Zunderschichten während des Schmiedens möglich ist [30-35]. Ein direkter Vergleich des Römischen Hammerschlags mit Literaturdaten ist jedoch nicht möglich, da die Schmiedebedingungen in der römischen Esse nicht bekannt sind.
Danksagung. Unser Dank gilt Herrn DI Werner Artner, vom Röntgenzentrum der TU Wien, für die XRD Messungen.

Funding. Open access funding provided by TU Wien (TUW).

Open Access Dieser Artikel wird unter der Creative Commons Namensnennung 4.0 International Lizenz veröffentlicht, welche die Nutzung, Vervielfältigung, Bearbeitung, Verbreitung und Wiedergabe in jeglichem Medium und Format erlaubt, sofern Sie den/die ursprünglichen Autor(en) und die Quelle ordnungsgemäß nennen, einen Link zur Creative Commons Lizenz beifügen und angeben, ob Änderungen vorgenommen wurden. 
Die in diesem Artikel enthaltenen Bilder und sonstiges Drittmaterial unterliegen ebenfalls der genannten Creative Commons Lizenz, sofern sich aus der Abbildungslegende nichts anderes ergibt. Sofern das betreffende Material nicht unter der genannten Creative Commons Lizenz steht und die betreffende Handlung nicht nach gesetzlichen Vorschriften erlaubt ist, ist für die oben aufgeführten Weiterverwendungen des Materials die Einwilligung des jeweiligen Rechteinhabers einzuholen.

Weitere Details zur Lizenz entnehmen Sie bitte der Lizenzinformation auf http://creativecommons.org/licenses/by/4.0/deed.de.

\section{Literatur}

1. Karl, S.: Das römerzeitliche Marmorsteinbruchrevier Spitzelofen in Kärnten, Montanarchäologische Forschungen. Mit Beiträgen von P. Bayer, M. Grabner, M. Hainzmann, R. Haubner, C.A. Hauzenberger, A.G. Heiss, K. Layr, D. Modl, W. Prochaska, S. Strobl, B. Toškan, E. Wächter, M. WeißI und S. Wiesinger, Fundberichte aus Österreich, Beiheft 1, Bundesdenkmalamt (Hrsg.), Horn: Ferdinand Berger \& Söhne Ges.m.b.H., 2021

2. Harl, F; Harl, O.: http://lupa.at (Bilddatenbank zu antiken Steindenkmälern) (März 2021)

3. Djurić, B.; Müller, H.W.: White Marbles in Noricum and Pannonia. An outline of the Roman Quarries and their Products, in Jockey, P. (Hrsg.): $\Lambda$ EYKO $\Sigma \Lambda \mid \Theta O \Sigma$. Marbres et autres roches de la Méditerranée antique. Études interdisciplinaires/Interdisciplinary Studies on Mediter ranean Ancient Marble and Stones. Éditions Karthala, Maison méd iterranéenne des sciences de l’homme, Paris, Aix-en-Provence, 2011, pp 111-127

4. Koželj, T.: Extraction of blocks in antiquity. Special methods of analysis, in Herz, N.; M. Waelkens, M (Hrsg.): Classical Marble. Geochemistry. Technology. Trade, NATO ASI Series E. Applied sciences, 153, Dordrecht-Boston-London: Kluwer Academic Publishers, 1988 pp 31-39

5. Bülow-Jacobsen, A.: Mons Claudianus. Ostraca graeca et latina IV. The Quarry-Texts. O. Claud, Kairo: Documents de Fouilles de I'Institut Français d'Archéologie Orientale, 47 (2009), pp 632-896

6. Straube, H.: Die Stadt auf dem Magdalensberg - Norischer Stahl, Ferrum Noricum und die Stadt auf dem Magdalensberg, Vienna: Springer, 1996

7. Furger, A.R.: Antike Stahlerzeugung - Ein Nachweis der Aufkohlung von Eisen aus Augusta Raurica. Beiträge zur Technikgeschichte, 2 Dr. h.c. Alfred-Mutz-Stiftung (Hrsg.), Basel, Frankfurt a. M.: LIBRUM Publishers \& Editors, 2019

8. Scheiblechner, W.; Karl, S.; Modl, D.; Strobl, S.; Haubner, R.: Untersuchung eines Doppelspitzschlägels aus dem römerzeitlichen Marmorsteinbruchrevier Spitzelofen in Kärnten, Österreich, Berg- und Hüttenmännische Monatshefte, 166 (2021) https://doi.org/10.1007/ s00501-021-01086-2

9. Haubner, R.: Schatz, I: Schatz, F; Scheiblechner, W.: Schubert, W.D.; Strobl, S.: Archaeometallurgical simulations of the processes in bloomery furnaces from the Hallstatt and medieval period. Materials Science Forum, 782 (2014), pp 641-644

10. Mutz, A.: Römisches Schmiedehandwerk. Auguster Monatshefte, 1, Römermuseum Augst, 1976

11. Dworakowska, A.: Quarries in Roman Provinces. Ossolineum, Wrocław 1983

12. Banti, L.: Antiche lavorazioni nelle cave lunensi. Studi Etruschi, 5, 1931, pp 475-497

13. Dolci, E.: Marmora lunensia. Quarrying technology and archaeological use, in Herz, N.; Waelkens, M. (Hrsg.): Classical Marble. Geochemistry. Technology. Trade, NATO ASI Series E. Applied sciences, 153, Dordrecht-Boston-London: Kluwer Academic Publishers, 1988, pp 77-84

14. Nicolini P.; Ozioso, S.: Cave e siti estrattivi. Introduzione alla carta di localizzazione e schedatura presenti nel DVD. Schedatura delle cave e dei siti in cui sono state rinvenute testimonianze di antiche attività estrattive nel bacino marmifero di Carrara, in Paribeni, E.; Segenni, S. (Hrsg.): Notae lapicidinarum dalle cave di Carrara, Pisa University Press, 2015, pp 97-104
15. Sodini, J.-P.; Lambraki, A.; Koželj, T.: Les carrières de marbre à I'époque paléochrétienne, in Aliki I. Études Thasiennes, 9, Paris: École Française d'Athènes, 1980, pp 81-146

16. Sapène, $B$ : Autels votifs, Atelier de Marbriers et Sanctuaire GalloRomains découverts à Saint-Béat (Hte-Gne) en 1946. Revue de Comminges, 59 (1946), pp 283-325

17. Peacock, D.P.S.; Maxfield, V.A.: Mons Claudianus. Survey and Excavation 1987-1993. Volume 1. Topography and Quarries. Kairo: Fouilles de I'Institut français d'archéologie orientale du Caire, 37 (1997)

18. https://www.youtube.com/watch?v=HtNLEYOnFRs (März 2021)

19. Melzer, W.: Schmiedehandwerk in Mittelalter und Neuzeit. Beiträge des 6. Kolloquiums des Arbeitskreises zur archäologischen Erforschung des mittelalterlichen Handwerks, Soester Beiträge zur Archäologie, 5, Soest: Westfälische Verlagsbuchhandlung Mocker \& Jahn, 2004

20. Dungworth, D.; Wilkes, R.: An Investigation of Hammerscale. Technology Report, Research Department Report, 26, English Heritage, Fort Cumberland, 2007

21. Dungworth, D.; Wilkes, R.: Understanding hammerscale: the use of high-speed film and electron microscopy. Historical Metallurgy, 43 (2009), pp 33-46

22. Khevenhüller, G.; Mitsche, R.; Trojer, F.: Aufbau dreier römischer Eisenluppen und der damit verbundenen Schlackenbestandteile vom Magdalensberg, Kärnten, Carinthia, II, 151 (1961), Nr. 71, S. 81-87

23. Strobl, S.; Haubner, R.; Klemm, S.: Metallographic investigations of a historical bloom found in Styria-Austria. in: Special Issue, 14th International Symposium on Metallography, Acta Metallurgica Slovaca Conference, 1 (2010), pp 655-660

24. Strobl, S.; Haubner, R.; Klemm, S.: Microstructure of a nail from the Modern Period found in the context of a historical road in Styria / Austria, in: Longeauerova, M. (Hrsg.): Special Issue, 14th International Symposium on Metallography, Acta Metallurgica Slovaca Conference, 1 (2010), pp 661-664

25. Strobl, S.; Scheiblechner, W.; Haubner, R.: Damaszenerschmieden mit Gusseisen und Stahl. in Petzow, G. (Hrsg.): Sonderbände der Praktischen Metallographie 47, INVENTUM GmbH, 2015, pp 127-132

26. Haubner, R.; Strobl S.: Copper-Coated Roman Coins - Subferrati; Römische Eisenmünzen mit Kupferbeschichtung - Subferrati, Praktische Metallographie - Practical Metallography, 53 (2016), pp 273-294

27. Klemm, S.; Strobl, S.; Haubner, R.: Mediaeval Iron Smelting in the Area of the Iron Mountain (Steirischer Erzberg) at Eisenerz, Styria (Austria), in: Mining in European History and its Impact on Environment and Human Societies, Innsbruck: Forschungszentrum HiMAT; innsbruck university press, 2013, pp 103-109

28. Shornikov, S.I.; Shornikova, M.S.: Thermodynamic Properties of the CaO-FeO Melts, Experiment in Geosciences, 25 (2019), pp 82-85

29. High Temperature Oxidation and Corrosion of Metals, Young D.J. (Editor), Second Edition 2016, Amsterdam et al.: Elsevier Ltd.

30. Tuck, C.W.; Odgers, M.; Sachs, K.: Die Verzunderungsgeschwindigkeit von reinem Eisen und Weichstahl in Sauerstoff, Wasserdampf und Kohlendioxid bei Temperaturen zwischen 850 und $1000^{\circ} \mathrm{C}$, Werkstoffe und Korrosion, 17 (1966), pp 34-48

31. Schmahl, N.G., Baumann, H., Schenck, H.: Die Temperaturabhängigkeit der Verzunderung von reinem Eisen in Sauerstoff, Archiv für das Eisenhüttenwesen, 29 (1958), pp 83-88 https://doi.org/10.1002/ srin. 195802678

32. Paidassi, J.: Oxidation of Iron in Air between 700 and $1250^{\circ} \mathrm{C}$, Rev. Met. LIV, 54 (1957), pp 569-585

33. Paidassi, J.: Sur la croissance en aiguilles de la phase hematite dans les pellicules d'oxydation du fer aux temperatures élévées (Über das Nadelwachstum der Hämatitphase in Eisenoxidfilmen bei erhöhten Temperaturen), Acta Metallurgica, 6 (1958), pp 778-780

34. Garnaud, G.; Rapp, R.A.: Thickness of the oxide layers formed during the oxidation of iron, Oxidation of Metals, 11 (1977), pp 193-198 https://doi.org/10.1007/BF00606543

35. Larsson, H.; Jonsson, T.; Naraghi, R.; Gong, Y.; Reed, R.C.; Ågren, J.: Oxidation of iron at $600^{\circ} \mathrm{C}$-experiments and simulations, Materials and Corrosion, 68 (2017), pp 133-142 https://doi.org/10.1002/maco. 201508781

Hinweis des Verlags. Der Verlag bleibt in Hinblick auf geografische Zuordnungen und Gebietsbezeichnungen in veröffentlichten Karten und Institutsadressen neutral. 